\title{
A Nonlinear Cable Bracing Inerter System for Vibration Control
}

\author{
Xinlei Ban 1,3,a, Songtao Xue 1,2,b , Jianfei Kang ${ }^{1, c}$, Kohju Ikago $^{3, d}$ and Liyu Xie ${ }^{1, e,{ }^{*}}$ \\ ${ }^{1}$ Department of Disaster Mitigation for Structures, Tongji University, Shanghai, China \\ ${ }^{2}$ Department of Architecture, Tohoku Institute of Technology, Sendai, Japan \\ ${ }^{3}$ International Research Institute of Disaster Science, Tohoku University, Sendai, Japan \\ a1610229@tongji.edu.cn, bxue@tongji.edu.cn, ckangjianfei@tongji.edu.cn, \\ dikago@irides.tohoku.ac.jp, eliyuxie@tongji.edu.cn
}

\begin{abstract}
Keywords: Nonlinear Cable Bracing, Inerter System, Bending-Shear Model, Vibration Mitigation, Optimization Design
\end{abstract}

\begin{abstract}
This study proposes a nonlinear cable model for the cable-bracing inerter system (CBIS). In a CBIS, cables are introduced to connect inerter systems and the structure for translation-to-rotation conversion. This CBIS employs an inerter element, a nonlinear cable bracing element and an additional damping element to utilize their synergy benefits. This paper aims to investigate the control effect of the nonlinear CBIS for high-rise buildings that are represented as bending-shear type models. First, a nonlinear inerter system is incorporated into a single-degree-of-freedom (SDOF) system and the mechanical model is proposed. An optimum design method is then developed for a high-rise building system equipped with a CBIS and the time-history analyses are conducted to validate the control effect of the CBIS. It is concluded that the employment of a CBIS can substantially improve the structural performance. A genetic algorithm can be used to obtain optimal parameters of a CBIS, thereby more effectively reducing the dynamic response of high-rise buildings.
\end{abstract}

\section{Introduction}

The inerter, a recently introduced mechanical element for structural control, has attracted an increasing amount of attention. It has been found to be effective because of their adjustable frequency, mass enhancement effect and damping enhancement principles [1,2]. Inerter is a twoterminal mechanical element [3] which generates a resisting force proportional to the relative acceleration between its two terminals. An apparent mass significantly larger than its physical one is obtained when the translational motion is converted into rotational one [4,5]. In the 1970s, Kawamata [6] developed a type of a fluid inerter designated liquid mass pump, which was a pioneering work applying an inerter in the field of civil engineering. In 1999, Arakaki et al. [7] used the ball screw mechanism to amplify the efficient output force of a viscous damper for suppressing structural vibrations, although the inertance yielded by the device was not used intentionally. Inerter systems have been demonstrated to be highly effective in mitigating both inter-story drifts and floor accelerations for low-rise and medium-rise buildings. Actually, this system is attracting especially for high-rise buildings.

Bracing systems are usually used in the implementation of the inerter system to convert translational movement into rotational one between the structure and the inerter system. In civil engineering field, researchers have developed various types bracing configurations [8] among which steel braces are the most used but few of them involve cables. However, the advantages of cable braces are numerous and incontestable [9]. As the cables are being used only in tension, they are much thinner and lighter than most steel braces. Thus, they have negligible inertia and 
they are particularly suitable for the systems in which great accelerations are applied. Furthermore, cables are less expensive than most conventional mechanical components when it is long enough and can be an alternative method for connecting inerters with the main structure.

However, cables involve two important characteristics to be considered seriously when they are applied to the high-rise buildings. One is the undesirable buckling in load-carrying elements [10]. Another is the unilaterality of actuation imposed by using cables. This unilaterality requires an unrestricted space in length. These two characteristics are attributed to the inability of cables to bear compression. Combined with compression-resistant materials [11], the effects of buckling and limitations of the stroke length can be reduced.

The previous study [12] has already proposed the mechanical model of the CBIS and the optimization design method to minimize the magnitudes of structural displacement responses [13]. In this paper, this nonlinear cable model is incorporated into a bending-shear model. First, the motion equations of the nonlinear inerter system are derived. Second, the optimal design problem to minimize the magnitudes of structural displacement responses is formulated to obtain a set of optimal designs for a nonlinear CBIS. Finally, the dynamic time-history analyses are conducted to validate the effectiveness of the CBIS.

\section{A nonlinear cable bracing inerter system}

\section{Nonlinear model of the cable braces}

A CBIS uses cable braces to connect inerter systems and main structures for translation-torotation conversion. The mechanical system of a CBIS consists of an additional damping element, an inerter element and an equivalent cable bracing element. Fig. 1 shows the layout of the CBIS and the nonlinear cable hysteresis in which the compressive stiffness of the buckling restrainer is several times as large as the tension stiffness.

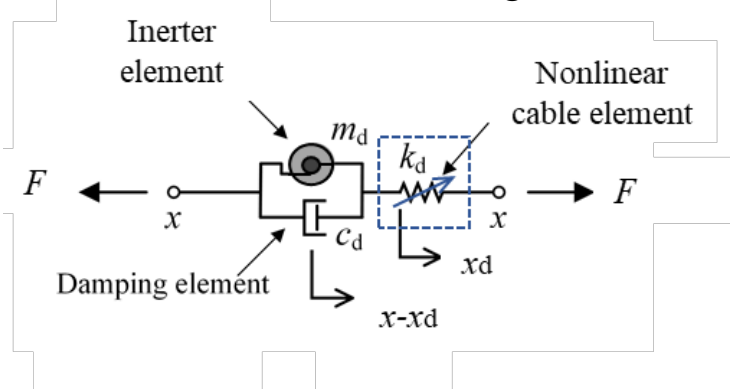

(a) CBIS

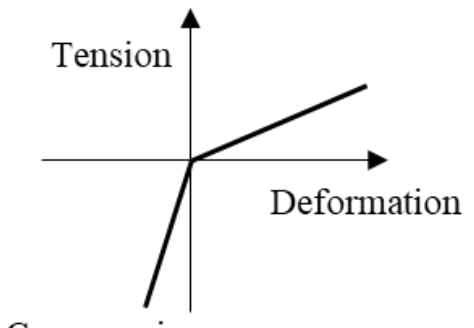

Compression

Fig. 1 Mechanical model of the nonlinear CBIS

In Fig.1a, $\boldsymbol{m}_{\mathrm{d}}$ and $\boldsymbol{c}_{\mathrm{d}}$ are the inertance and the damping coefficient of the CBIS. $\boldsymbol{k}_{\mathrm{d}}$ is the equivalent stiffness of the nonlinear cables which is given by Eq. 1:

$$
\left\{\begin{array}{l}
k_{\mathrm{d}}=k_{\mathrm{T}}, x_{\mathrm{d}}>0 \\
k_{\mathrm{d}}=k_{C}, x_{\mathrm{d}}<0
\end{array}\right.
$$

where $k_{\mathrm{T}}$ and $k_{\mathrm{C}}$ are the tension and compressive stiffnesses of the cable bracing element, respectively. The output force $F$ generated by the CBIS contains two main parts and can be calculated by the following equation:

$$
F=k_{\mathrm{d}} x_{\mathrm{d}}(t)=m_{\mathrm{d}}\left(\ddot{x}_{\mathrm{d}}(t)-\ddot{x}(t)\right)+c_{\mathrm{d}}\left(\dot{x}_{\mathrm{d}}(t)-\dot{x}(t)\right)
$$


where $\boldsymbol{x}(\boldsymbol{t})$ and $\boldsymbol{x}_{\mathrm{d}}(\boldsymbol{t})$ donate the displacement of the primary structure relative to the ground and displacement of the cable brace, respectively. Superimposed dots indicate derivatives with respect to the time.

\section{An SDOF structure equipped with a nonlinear CBIS}

Fig. 2 shows the analysis model of a CBIS-equipped SDOF structure. According to the force equilibrium conditions and the layout of the system, the equation of motion for the controlled structure can be represented as:

$$
m \ddot{x}(t)+c \dot{x}(t)+k x(t)+F=-m \ddot{x}_{g}(t)
$$

where $m, c$ and $k$ denote the mass, damping coefficient and stiffness of the primary structure, respectively; $\ddot{x}_{g}(t)$ is the acceleration of ground motion.

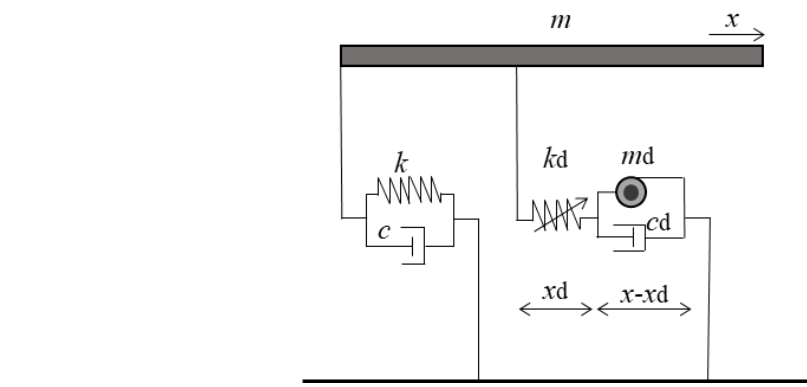

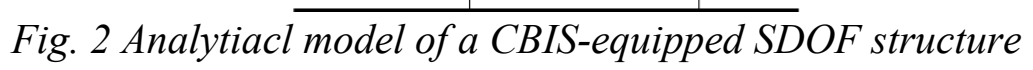

\section{Optimization design of a CBIS controlled multi-degree-of-freedom (MDOF) structure}

\section{Building model}

We reproduced an analytical model of the Osaka Prefectural Goverment Building at Sakishima, Osaka [14] from the published data. This 53-storey building has a height of $256 \mathrm{~m}$. The total mass of the building is 78,008 tons, and the characteristics for the analytical model are shown in Table 1. The first three natural angular frequencies are $1.257,3.734$ and $6.367 \mathrm{rad} / \mathrm{s}$, respectively.

In high-rise buildings, the bending deformation cannot be ignored and usually the bending deformation of the higher layer accounts for the a large proportion (as shown in Fig.3). In the $1^{\text {st }}$ mode of this structure, the components of shear deformation and bending deformation are similar when the floors are lower than the $43^{\text {rd }}$ floor. The proporation of bending deformation gradually increases when the floors are higher than the $43^{\text {rd }}$ floor. In the $2^{\text {nd }}$ and $3^{\text {rd }}$ modes, the bending deformation dominates in the floors above the $43^{\text {rd }}$ floor. In Fig.4, bending deformation is the same as shear deformation on the top floor. Therefore, the inerter systems are suggested to be installed vertically on the top of the building to make full use of the vertical component of the bending deformation so that the efficiency of the CBIS can be maximized (as shown in Fig.6).

Table 1 Fundamental periods of the analytical model

\begin{tabular}{|c|c|c|c|}
\hline Mode & $1^{\text {st }}$ & $2^{\text {nd }}$ & $3^{\text {rd }}$ \\
\hline Period [s] & 5.00 & 1.68 & 0.99 \\
\hline Angular frequency & 1.257 & 3.734 & 6.367 \\
\hline
\end{tabular}



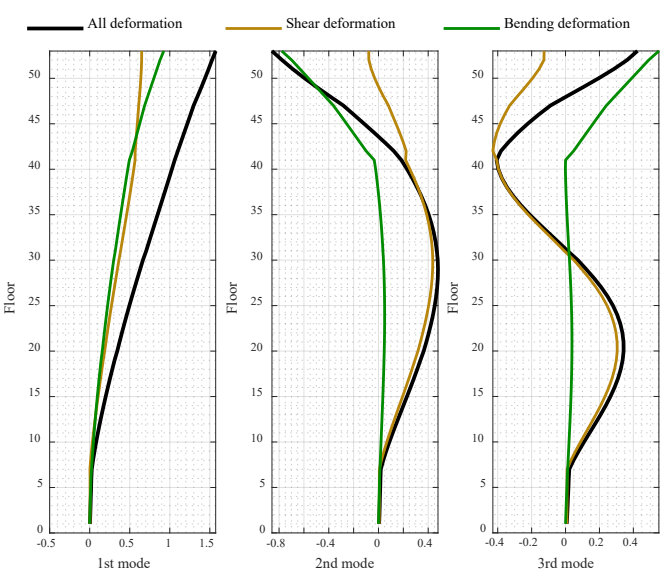

Fig.3 Participation mode vectors of theuncontrolled structure

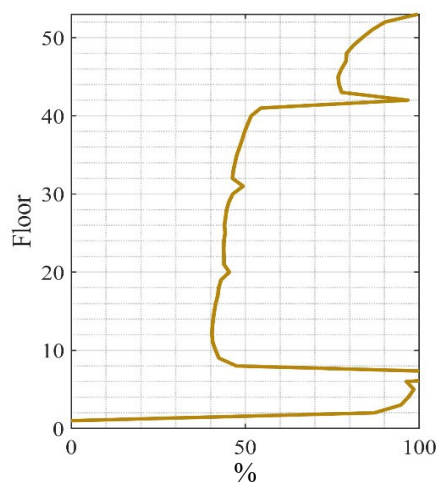

Fig.4 Ratios of bending deformation and shear deformation

\section{Uncontrolled building}

Considering this 53-storey bending-shear model shown in Fig.5, every storey of this model consists of a rotational and shear springs. These two springs are connected in series. Let $k_{\mathrm{sj}}$ and $k_{\mathrm{bj}}$ denote the shear stiffness and bending stiffness, respectively. Similarly, let $c_{\mathrm{sj}}$ and $c_{\mathrm{bj}}$ denote the damping coefficient of the dashpots arranged in shear and rotational directions, respectively. These two dashpots are also connected in series. The floor mass and its rotational mass moment of inertia are denoted by $m_{\mathrm{j}}$ and $J_{\mathrm{j}}$, respectively.

In this model, every storey has one translational and one rotational degree of freedom. Therefore, the total number of DOF should be 106 (53 in translation and 53 in rotation). We assume that the damping matrix $\mathbf{C}_{\mathrm{s}}$ for the primary structure is proporational to the stiffness matrix and the inherent damping of the $1^{\text {st }}$ mode of the structure equals $0.02, \mathbf{C}_{\mathrm{s}}$ can be obtained as follows:

$$
\mathbf{C}_{\mathrm{s}}=\frac{2 * 0.02}{{ }_{1} \omega_{\mathrm{p}}} \mathbf{K}_{\mathrm{s}}
$$

where ${ }_{1} \omega_{\mathrm{p}}$ is the first natural angular frequency of the primary structure. The equation of motion for the uncontrolled structure can be written as :

$$
\mathbf{M}_{\mathrm{s}} \ddot{\mathbf{X}}_{\mathrm{s}}+\mathrm{C}_{\mathrm{s}} \dot{\mathbf{X}}_{\mathrm{s}}+\mathbf{K}_{\mathrm{s}} \mathbf{X}_{\mathrm{s}}=-\mathbf{M}_{\mathrm{s}} \boldsymbol{\Gamma}_{\mathrm{s}} \ddot{x}_{g}
$$

where $\mathbf{M}_{\mathrm{s}}, \mathbf{C}_{\mathrm{s}}$ and $\mathbf{K}_{\mathrm{s}}$ are the structural mass, damping and stiffness matrixs of the primary structure, respectively. $\mathbf{X}_{\mathrm{s}}$ is the structural deformation vector. $\boldsymbol{\Gamma}_{\mathrm{s}}$ is a vector with entries equal to unity for translation DOFs and zreo for the others. $\mathbf{X}_{\mathrm{s}}$ consists of displacements and rotational angles defined as:

$$
\mathbf{X}_{\mathrm{s}}=\left[\begin{array}{llllllll}
x_{1} & x_{2} & \ldots & x_{N} & \theta_{1} & \theta_{2} & \ldots & \theta_{N}
\end{array}\right]^{\mathrm{T}}
$$

where $x j$ and $\theta j$ are the displacement and rotational angles relative to the ground, respectively. The mass matrix $\mathbf{M}_{\mathrm{s}}$ is defined as : 


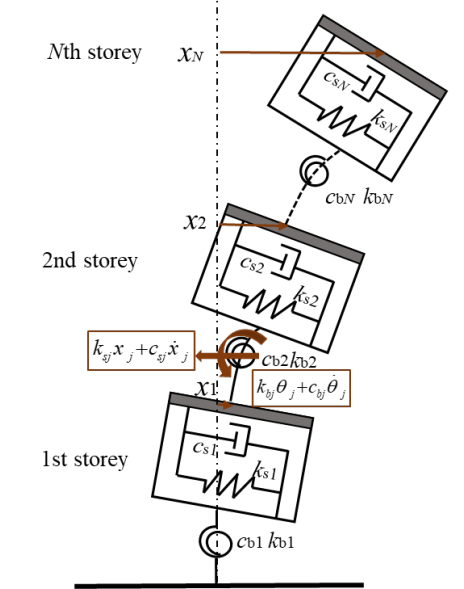

Fig. $\overline{5 \text { A bending-shear model }}$

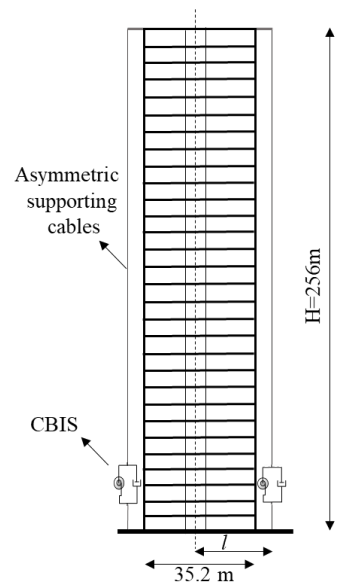

Fig.6 Installation location

$$
\mathbf{M}_{\mathrm{s}}=\operatorname{diag}\left[m_{1} m_{2} \ldots m_{N} J_{1}, J_{2} \ldots J_{N}\right]
$$

The influence coefficient vector $\boldsymbol{\Gamma}_{\mathrm{s}}$ is given by



Building with nonlinear CBIS

Based on the bending-shear model, the equation of motion for the building model with two CBISs under ground motion excitations as illtstrated in Fig.6 is derived as Eq.9.

$$
\mathbf{M} \ddot{\mathbf{X}}+\mathbf{C} \dot{\mathbf{X}}+\mathbf{K X}=-\mathbf{M \Gamma} \ddot{x}_{g}
$$

where

$$
\begin{aligned}
& \mathbf{M}=\left[\begin{array}{cc}
\mathbf{M}_{\mathrm{s}}+\mathbf{T M}_{\mathrm{d}} \mathbf{T}^{\mathrm{T}} & \mathbf{T M}_{\mathrm{d}} \\
\mathbf{M}_{\mathrm{d}} \mathbf{T}^{\mathrm{T}} & \mathbf{M}_{\mathrm{d}}
\end{array}\right], \mathbf{C}=\left[\begin{array}{cc}
\mathbf{C}_{\mathrm{s}}+\mathbf{T C}_{\mathrm{d}} \mathbf{T}^{\mathrm{T}} & \mathbf{T} \mathbf{C}_{\mathrm{d}} \\
\mathbf{C}_{\mathrm{d}} \mathbf{T}^{\mathrm{T}} & \mathbf{C}_{\mathrm{d}}
\end{array}\right], \mathbf{K}=\left[\begin{array}{cc}
\mathbf{K}_{\mathrm{s}} & \mathbf{0}_{2 N \times 2} \\
\mathbf{0}_{2 \times 2 N} & \mathbf{K}_{\mathrm{d}}
\end{array}\right],
\end{aligned}
$$

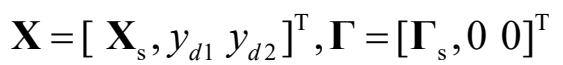

$$
\begin{aligned}
& \mathbf{M}_{\mathrm{d}}=\left[\begin{array}{cc}
m_{\mathrm{d}} & 0 \\
0 & m_{\mathrm{d}}
\end{array}\right], \mathbf{C}_{\mathrm{d}}=\left[\begin{array}{cc}
c_{\mathrm{d}} & 0 \\
0 & c_{\mathrm{d}}
\end{array}\right], \mathbf{K}_{\mathrm{d}}=\left[\begin{array}{cc}
k_{\mathrm{d}} & 0 \\
0 & k_{\mathrm{d}}
\end{array}\right], \quad \mathbf{T}=\left[\begin{array}{cc}
0 & 0 \\
0 & 0 \\
0 & 0 \\
\ldots & \ldots \\
\frac{l}{2} & -\frac{l}{2}
\end{array}\right]_{2 N \times 2}
\end{aligned}
$$

$\mathbf{T}$ is an installation matrix, denoting the installation location of the CBIS. Let $l$ be the distance from the center of the core to the tips of cables. $\mathbf{T}$ transforms the relative displacement vector into the inter-story drift vector. 
Optimization design problem

A numerical optimization method, genetic algorithm (GA), is used to design the inerter system. Because the inerter systems are installed vertically and only the bending deformation of the structure can be used, the mode with the largest bending deformation should be considered. As shown in Figs. 3 and 4, the optimization design target is the $1^{\text {st }}$ mode.

Herein, the effective inertance-mass ratio $\mu$, supporting spring stiffness ratio $\kappa$, and damping ratio of an inerter system $\xi$ are defined as:

$$
\mu=\frac{M_{\mathrm{d}}}{M_{\mathrm{s}}}=\frac{\phi^{\mathrm{T}} \mathbf{T}^{\mathrm{T}} \mathbf{M}_{\mathrm{d}} \mathbf{T} \phi}{\phi^{\mathrm{T}} \mathbf{M}_{\mathrm{s}} \phi}, \kappa=\frac{K_{\mathrm{d}}}{K_{\mathrm{s}}}=\frac{{ }_{1} \phi^{T} \mathbf{T}^{T} \mathbf{K}_{\mathrm{d}} \mathbf{T}_{1} \phi}{{ }_{1} \phi^{T} \mathbf{K}_{\mathrm{s} 1} \phi}, \xi=\frac{C_{\mathrm{d}}}{2 M_{\mathrm{s}} \omega_{\mathrm{r}}}=\frac{{ }_{1} \phi^{T} \mathbf{T}^{T} \mathbf{C}_{\mathrm{d}} \mathbf{T}_{1} \phi}{2{ }_{1} \phi^{T} \mathbf{M}_{\mathrm{s} 1} \phi \omega_{\mathrm{r}}}
$$

The inertance-mass ratio $\mu$ is set to 0.1 . The optimization design problem can be expressed mathematically to purse the optimal solutions:

$$
\begin{array}{cr}
\text { find } & \boldsymbol{y}=\{\xi, \kappa, \gamma\} \\
\text { to minimize } & \sum_{i=1}^{106} x_{i}^{\max }(\boldsymbol{y}) \\
\text { subject to } \quad \kappa=\frac{(1-\sqrt{1-4 \mu})^{2}}{2(1+\gamma) \mu}
\end{array}
$$

where the subscript $i$ denotes the $i$-th storey. $\boldsymbol{y}$ is the variable vector containing $\xi$, $\kappa$ and compression-tension stiffness ratio $\gamma$. The sum of the maximum displacement response of the top floor is to be minimized. The average of the compressive and tension stiffnesses of cable braces is equal to the supporting spring stiffness $k_{\mathrm{d}}$, and the stiffness ratio $\kappa$ is constrained to the optimum stiffness obtained from the fixed point method [17]. Using genetic algorithm, we employed an artificial earthquake BCJ-L2 as the input ground motion and obtained a set of optimal parameters listed in Table 2.

Table 2 Optimal design

\begin{tabular}{|l|c|c|c|}
\hline \multicolumn{4}{|c|}{$\mu=0.1$} \\
\hline & $\kappa$ & $\xi$ & $\gamma$ \\
\hline Constrained range & {$[0.01,1]$} & {$[0.01,1]$} & {$[1,20]$} \\
\hline Optimal parameters & 0.231 & 0.035 & 1.118 \\
\hline
\end{tabular}

To verify the optimal parameters of the nonlinear CBIS, dynamic time-history analyses are conducted in the time domain based on the Newmark's $\beta$ method $(\beta=1 / 4)$ as shown in Fig.7 and the vibration reduction effects are listed in Table 3 . Note that these results are based on the reproduced analytical model and thus they may not accurately represent the performance of the building we referred to.

Table 3 Comparison of structural responses

\begin{tabular}{|c|c|c|c|c|c|c|}
\hline \multirow{2}{*}{} & \multicolumn{2}{|c|}{$\begin{array}{c}\text { Primary } \\
\text { structure }\end{array}$} & \multicolumn{2}{c|}{$\begin{array}{c}\text { Controlled } \\
\text { structure }\end{array}$} & \multicolumn{2}{c|}{$\begin{array}{c}\text { Reduction effects } \\
{[\%]}\end{array}$} \\
\cline { 2 - 7 } & Peak & RMS & Peak & RMS & Peak & RMS \\
\hline Inter-story drift angle & $1 / 62$ & $1 / 165$ & $1 / 133$ & $1 / 468$ & 53.38 & 64.74 \\
\hline Absolut acceleration $\left[\mathrm{m} / \mathrm{s}^{2}\right]$ & 6.87 & 1.91 & 5.67 & 1.27 & 17.48 & 33.51 \\
\hline Displacement $[\mathrm{m}]$ & 2.12 & 0.96 & 0.90 & 0.33 & 57.55 & 65.63 \\
\hline
\end{tabular}




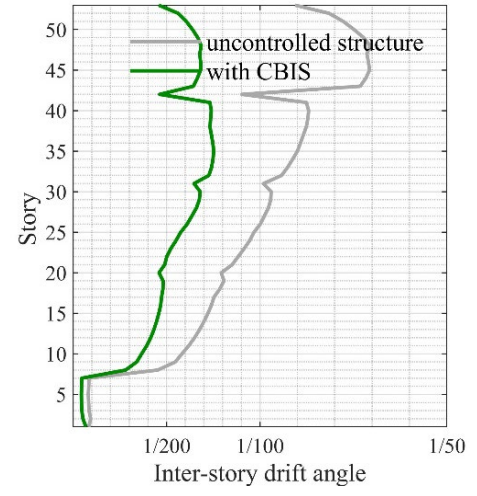

(a) Maximum inter-story drift angle

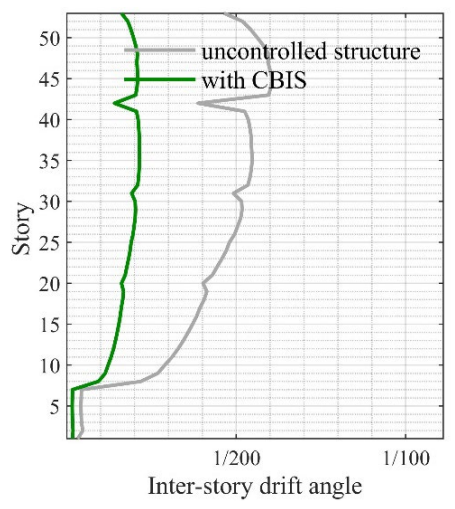

(b) RMS inter-story drift angle

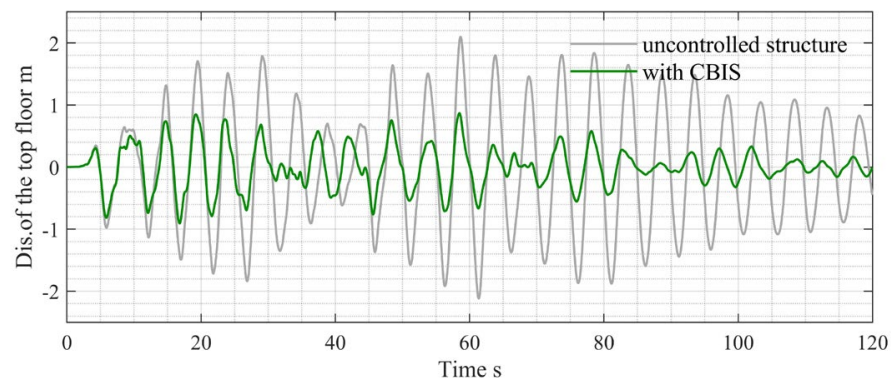

(c) Displacement of the $53^{\text {rd }}$ floor

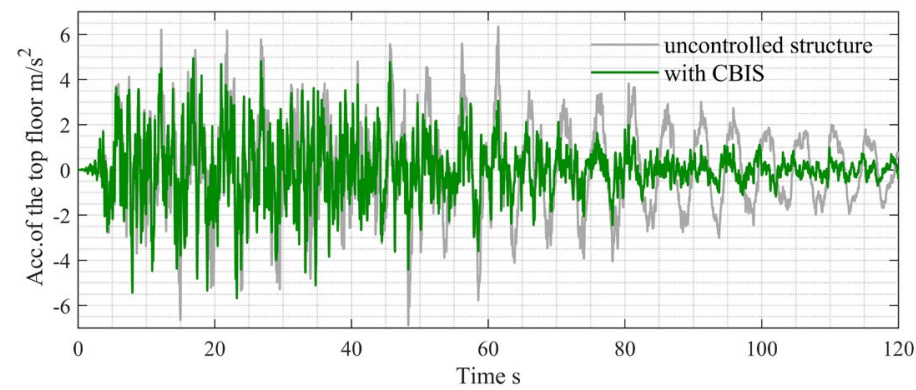

(d) Acceleration of the $53^{\text {rd }}$ floor

Fig.7 Time history of the 53-storey structure under BCJ-L2

With the optimal parameters, the displacement responses, the acceleration responses and the inter-story drift angles of the primary structure are all well mitigated by the CBIS with asymmetric supporting spring stiffness. This means that when the inerter systems are installed vertically, we can obtain the effect of suppressing overall bending deformation and shear deformation of high-rise buildings.

\section{Conclusions}

This study proposes a nonlinear cable bracing element which can avoid buckling in tension-only cables and thus exploit the damping forces in compression. Dynamic characteristics of a highrise building incorporated with a novel asymmetric inerter system are examined. A genetic algorithm (GA) was used to determine the parameters of CBISs so as to minimize structural displacement responses. Then, the vibration mitigation effect of the CBIS is evaluated under a synthetic ground motion BCJ-L2 provided by the Building Center of Japan. The most inportant findings are summarized as follows: 
(1) Genetic algorithm was applied to seismic response analysis of the CBIS-equipped bendingshear building model. With this method, optimum designs of the CBIS can be obtained to minimize the maximum displacement response of the top floor.

(2) Based on the optimal parameters, the CBIS not only reduces the displacement response and the acceleration response but also significantly mitigates the maximum inter-story drift by $53.38 \%$.

(3) It is noteworthy to point out that in terms of reducing seismic acceleration responses, the control effect is not as good as the displacement responses. One possible reason is that the position where the CBISs are placed maybe ineffective for controlling the seismic acceleration responses. To pursue better performance, the position optimal design under earthquake and multi-modal control should be the next step of research.

\section{Acknowledgements}

This study was supported by the National Natural Science Foundation of China (Grant No.51778490 and 51978525), Open Research Fund Program of Guangdong Key Laboratory of Earthquake Engineering and Application Technology (Grant No. 2017B030314068), the Natural Science Foundation of Shanghai (Grant No. 20ZR1461800). The first author also would like to gratefully acknowledges the support of the China Scholarship Council by the joint PhD program.

\section{References}

[1] K. Ikago, K. Saito, N. Inoue, Seismic control of single-degree-of-freedom structure using tuned viscous mass damper, Earthq. Eng. Struct. Dyn. 41 (2012) 453-474.

https://doi.org/10.1002/eqe.1138

[2] R.F. Zhang, Z.P. Zhao, C. Pan, K. Ikago, S.T. Xue, Damping enhancement principle of inerter system, Struct. Control Health Monit. (2020). https://doi.org/10.1002/stc.2523

[3] M.Z.Q. Chen, Y. Hu, L. Huang, G. Chen, Influence of inerter on natural frequencies of vibration systems, J. Sound. Vib. 333 (2014) 1874-1887.

https://doi.org/10.1016/j.jsv.2013.11.025

[4] Z.P. Zhao, R. F. Zhang, Z. Lu, A particle inerter system for structural seismic response mitigation, J. Frankl. Inst. 356 (2019) 7669-7688. https://doi.org/10.1016/j.jfranklin.2019.02.001

[5] Q.J. Chen, Z.P. Zhao, R.F. Zhang, C. Pan, Impact of soil-structure interaction on structures with inerter system, J. Sound. Vib. 433 (2018) 1-15. https://doi.org/10.1016/j.jsv.2018.07.008

[6] S. Kawamata, Development of a vibration control system of structures by means of mass pumps. Institute of Industrial Science, University of Tokyo, Tokyo, Japan, 1973.

[7] T. Arakaki, H. Kuroda, F. Arima, Y. Inoue, K. Baba, Development of seismic devices applied to ball screw: Part 1 basic performance test of rd-series, J. Archit. Build. Sci. 5 (1999) 239-244. https://doi.org/10.3130/aijt.5.239_1

[8] J.S. Hwang, J. Kim, Y.M. Kim, Rotational inertia dampers with toggle bracing for vibration control of a building structure, Eng. Struct. 29 (2007) 1201-1208. https://doi.org/10.1016/j.engstruct.2006.08.005 
[9] S.E. Landsberger, A minimal, minimal linkage: the tension-compression parallel link manipulator, Robotics Mechatron Manufact. Syst. 2 (1993) 81-88. https://doi.org/10.1016/B9780-444-89700-8.50017-8

[10] M. Kurata, R.T. Leon, R. DesRoches, Rapid seismic rehabilitation strategy: concept and testing of cable bracing with couples resisting damper, J. Struct. Eng. 138 (2012) 354-362. https://doi.org/10.1061/(ASCE)ST.1943-541X.0000401

[11] H. Kida, K. Kakemoto, N. Hori, K. Ikago, N. Inoue, Control of bending behavior of high rise buildings by tuned viscous mass dampers installed in the vertical direction by support members with nonlinear stiffness properties. Part 3. Study on 3D frame model, AIJ Annual Convention (2020).

[12] L.Y. Xie, X.L. Ban, S.T. Xue, K. Ikago, J.F. Kang, H.S. Tang, Theoretical study on a cablebracing inerter system for seismic mitigation, Appl. Sci. 9 (2019) 4096. https://doi.org/10.3390/app9194096

[13] X.L. Ban S.T. Xue, K. Ikago, Optimal design of a cable-bracing inerter system containing asymmetric supporting spring stiffness, AIJ Annual Convention (2020).

[14] T. Itou, Feasibility study on a tuned hydraulic inerter-damper with large apparent mass incorporated into super high-rise structure, Tohoku University (2019). 